\title{
A SEVEN YEAR CAMPAIGN ON WR22
}

\author{
G. RAUW* J.-M. VREUX, E. GOSSET,* D. HUTSEMEKERS, \\ P. MAGAIN**, J. MANFROID*** and M. REMY \\ Institut d'Astrophysique, Université de Liège, Liège, Belgium \\ and \\ K. ROCHOWICZ \\ Institute of Astronomy, Nicolaus Copernicus University, Torun, Poland
}

\begin{abstract}
New spectroscopic and photometric observations of WR22 are presented. High signal-to-noise digital spectra with high dispersion are used to improve the parameters of the orbit $(P=80.31 \pm 0.01 \mathrm{~d})$, formerly determined on the basis of photographic spectra only. The intrinsic photometric variability of WR22 and recent observations of the eclipse are also discussed.
\end{abstract}

Key words: stars: Wolf-Rayet - binaries - WR22

WR22 (HD 92740) is a bright $(V \approx 6.4)$ Wolf-Rayet star of spectral type WN7 and a member of the Car OB1 association. It is single-lined (SB1, van der Hucht et al. 1981) with a period of 80.35 days (Moffat \& Seggewiss 1978; Conti et al. 1979). Since 1989, we have known that WR22 is an eclipsing binary (Balona et al. 1989; Gosset et al. 1991) with only one eclipse (corresponding to the WR star in front of the companion).

Photometric variability has been reported by several authors (see e.g., Moffat \& Seggewiss 1978; Balona et al. 1989; Gosset et al. 1991, 1994). In the framework of the Long-Term Photometry of Variables project (LTPV, Sterken 1983), a total of 218 observations were obtained between JD 2447228 and JD 2449182, with the Danish $50 \mathrm{~cm}$ telescope at ESO equipped with its four channel Strömgren photometer. The computed standard deviations are well above the observational errors: $\sigma_{u, v, b, y}=0.017,0.011,0.010,0.010$. The power spectra, computed using Deeming's (1975) method, of the $v, b$ and $y$ filter data are similar, whereas the $u$ data display a quite different spectrum. The highest peak in the $b$ spectrum is located at $\nu=0.0199 d^{-1}$, whereas no significant peak is found at $\nu=0.0124 d^{-1}$ i.e., at the frequency corresponding to the orbital motion (see Fig. 1).

During our campaign, the eclipse has been observed on several occurrences in order to progressively fill the daily gaps in the folded light-curve and to reduce the dispersion due to the intrinsic variability of the WR star. Investigation of the light-curve still goes on, but the presently available data indicate that the orbital period of WR22 should be $P=80.31( \pm 0.01)$ days, a value independently confirmed by our radial velocity curve. Most of our

* Aspirant au Fonds National de la Recherche Scientifique

** Chercheur Qualifié au Fonds National de la Recherche Scientifique

*** Directeur de Recherches au Fonds National de la Recherche Scientifique 


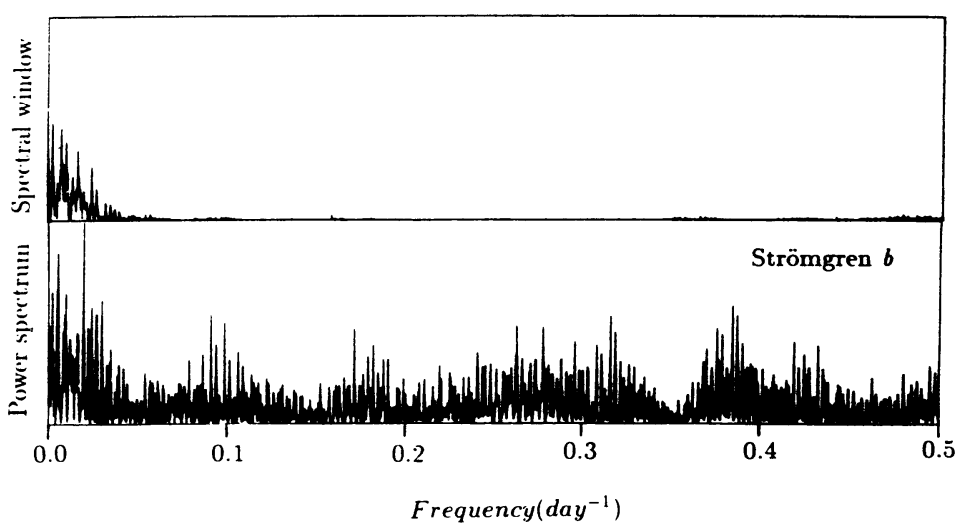

Fig. 1. Power spectrum of our LTPV data on WR22

spectroscopic data were obtained between 1987 and 1992 at the ESO Coudé Auxiliary Telescope (CAT) with a resolution of $0.07 \AA$. Some additional spectra were acquired in 1986-1987 with the B\&C Cassegrain Spectrograph at the $2.2 \mathrm{~m} \mathrm{ESO/MPI} \mathrm{telescope} \mathrm{(resolution} \mathrm{of} 0.64 \AA$ ). A new orbital solution has been derived with the program of Wolfe, Horak \& Storer (1967), using the radial velocities of the narrow emission line $\mathrm{N}$ IV $\lambda 4058$, which is known to form in the deeper layers of the WR stellar wind, thus reflecting the actual orbital motion of the WR star. We find that eclipse and periastron nearly coincide and that the resulting dates are in rather good agreement with those of the observed eclipses.

\section{Acknowledgements}

This research was supported in part by contract ARC90/94-140 'Action de recherche concertée de la Communauté Française' (Belgium) and by contract SC005 of the Belgian Programme Service Centres and Research Networks. Our thanks also go to J.-P. Swings for having read and significantly improved a first draft of this paper.

\section{References}

Balona, L.A., Egan, J., Marang, F. 1989, MNRAS 240, 103

Conti, P.S., Niemela, V.S., Walborn, N.R. 1979, ApJ 228, 206

Deeming, T.J. 1975, Ap Space Sci. 36, 137

Gosset, E., Remy, M., Manfroid, J., Vreux, J.-M., Balona, L.A., Sterken, C., Franco, G.A.P. 1991, Inf. Bull. Var. Stars No. 3571, p. 1

Gosset, E., Rauw, G., Manfroid, J., Vreux, J.-M., Sterken, C. 1994, in: C. Sterken \& M.J.H. de Groot (eds.) The Impact of Long-Term Monitoring on Variable Star Research, NATO ASI Series Vol. C 436 (Dordrecht: Kluwer), p. 101

Moffat, A.F.J., Seggewiss, W. 1978, $A \&$ \& 70, 69

Sterken, C. 1983, The Messenger 33, 10

van der Hucht, K.A., Conti, P.S., Lundström, I., Stenholm, B. 1981, Space Science Reviews 28, 227

Wolfe, R.H., Horak, H.G., Storer, N.W. 1967, in: M. Hack (ed.), Modern Astrophysics (New York: Gordon \& Breach), p. 251 